\title{
Optimal Robust Control for Uncertain Impulsive Systems*
}

\author{
Liu Bin ${ }^{1,2}$, Hill David J. ${ }^{2}$ \\ 1. Department of Information and Computation Sciences, Hunan University of Technology, Zhuzhou 412008, P. R. China. \\ E-mail: oliverliu78@163.com \\ 2. Department of Information Engineering, Australian National University, ACT 0200, Australia. \\ E-mail: David.Hill@anu.edu.au
}

\begin{abstract}
This paper aims to study the optimal robust control problem for uncertain impulsive systems. By using the Lyapunov function method and algebraic inequalities, conditions are derived under which not only the uncertain impulsive system is robustly asymptotically stable and the optimal bound of the hybrid performance functional can be estimated. The results are then specialized to interval linear impulsive systems with quadratic hybrid performance functional. Riccati inequality conditions are used to design feedback controllers to stabilize asymptotically the interval linear impulsive system and the optimal bound of the quadratic hybrid performance functional are derived.
\end{abstract}

Key Words: Uncertain impulsive systems, Hybrid performance functional, Robust stability, Riccati inequality, Interval matrix

\section{INTRODUCTION}

Impulsive systems arise in many applied fields such as control technology, communication networks, and biological management. Since impulsive systems provide a natural framework for mathematical modelling of many physical phenomena, their study is assuming a greater importance. The stability theory of impulsive systems and impulsive control have been developed quite rapidly in recent years. See $[1-4,7,10-13,16-19]$ and references cited therein.

The stability of a system may be affected by many factors including medelling uncertainty. With respect to uncertainty in the system, robust stability analysis is a very important research topic. Many results have been published to investigate the robust stability problem for uncertain continuous systems, (see e.g. [5]). However, there are few stability results reported for impulsive systems with uncertainties and disturbance inputs, (see e.g. [6], [9-10, 14-15]).

In this paper, we study the problem of robust stability and optimal feedback control for uncertain impulsive systems with a hybrid performance functional. Since there exist uncertainties in the system, it is difficult to calculate explicitly the value of the hybrid performance functional. Hence, it is meaningful to estimate the bound of the performance function. By employing the results developed in the [9], we derive some criteria under which the uncertain impulsive system is robustly asymptotically stable and the bounds of the hybrid performance functional can be estimated. These criteria are shown to be less conservative than those in [6].

The rest of this paper is organized as follows. In Section 2, we make some preliminaries and introduce some notations. We state and prove our main results in Sections 3. In Section 4 , we discuss the problem for interval linear impulsive systems with quadratic hybrid performance functional.

\section{PRELIMINARIES}

Let $\mathbf{R}^{n}$ denote the $n$-dimensional Euclidean space. Let $\mathbf{R}_{+}=[0,+\infty), \mathbf{N}=\{1,2, \cdots\}$. Denote by $\mathcal{K}$ the class of functions $\phi: \mathbf{R}_{+} \rightarrow \mathbf{R}_{+}$, which are continuous, strictly increasing and $\phi(0)=0, \mathcal{K}_{0}$ the class of continuous functions $\psi: \mathbf{R}_{+} \rightarrow \mathbf{R}_{+}$such that $\psi(s)=0$ if and only if

\footnotetext{
* This work is supported by the Australian Research Council.
}

$s=0$, and $P C$ the class of functions $\lambda: \mathbf{R}_{+} \rightarrow \mathbf{R}_{+}$, where $\lambda$ is continuous everywhere except $t_{k}(k \in \mathbf{N})$ at which $\lambda$ is left continuous and the right limit $\lambda\left(t_{k}^{+}\right)$exists. Let $\|\cdot\|$ stand for the Euclidean norm in $\mathbf{R}^{n}$. Let $\mathrm{P}^{n \times n}$ denote the set of $n \times n$ positive definite matrices, and $\mathbf{N}^{n \times n}$ the set of nonnegative definite matrices.

Consider the general uncertain impulsive controlled systems in form

$$
\begin{aligned}
& \dot{x}(t)=\tilde{f}_{c}\left(t, x(t), u_{c}(t)\right), \quad t \in\left(t_{k-1}, t_{k}\right] \\
& \Delta x=\tilde{f}_{d}\left(t, x(t), u_{d}(t)\right), \quad t=t_{k}, k \in \mathbf{N} \\
& x\left(t_{0}^{+}\right)=x_{0}
\end{aligned}
$$

where $0<t_{0}<t_{1}<t_{2}<\cdots<t_{k}<\cdots, t_{k} \rightarrow \infty$ as $k \rightarrow \infty$, and $x \in \mathbf{R}^{n}, \Delta x=x\left(t_{k}^{+}\right)-x\left(t_{k}^{-}\right)=x\left(t_{k}^{+}\right)-x\left(t_{k}\right)$; $u_{c}: \mathbf{R}_{+} \rightarrow \mathrm{U}_{c} \subset \mathbf{R}^{n_{c}}, u_{d}: \mathbf{R}_{+} \rightarrow \mathrm{U}_{d} \subset \mathbf{R}^{n_{d}}$, with $u_{c}(0)=0, u_{d}(0)=0$, here $\left(\mathrm{U}_{c}, \mathrm{U}_{d}\right) \subset \mathbf{R}^{n_{c}} \times \mathbf{R}^{n_{d}}$ is the class of admissible hybrid control inputs; $\tilde{f}_{c} \in \tilde{F}_{c}=\left\{\tilde{f}_{c}\right.$ : $\left.\mathbf{R}_{+} \times \mathbf{R}^{n} \times \mathbf{R}^{n_{c}} \rightarrow \mathbf{R}^{n}, \tilde{f}_{c}(t, 0,0) \equiv 0\right\}$, where $\tilde{f}_{c}(t, x, y)$ is Lipschitz continuous in $(x, y), \tilde{f}_{d} \in \tilde{F}_{d}=\left\{\tilde{f}_{d}: \mathbf{R}_{+} \times\right.$ $\left.\mathbf{R}^{n} \times \mathbf{R}^{n_{d}} \rightarrow \mathbf{R}^{n}, \tilde{f}_{d}(t, 0,0) \equiv 0\right\}$, where $\tilde{f}_{d}$ is continuous. $\tilde{F}_{c}$ and $\tilde{F}_{d}$ denote the set of nonlinear uncertain functions with $\tilde{f}_{c 0} \in \tilde{F}_{c}$, and $\tilde{f}_{d 0} \in \tilde{F}_{d}$ defining the nominal nonlinear functions of $\tilde{f}_{c} \in \tilde{F}_{c}$ and $\tilde{f}_{d} \in \tilde{F}_{d}$, respectively. Denote $\tilde{F}=\tilde{F}_{c} \times \tilde{F}_{d}$.

The hybrid performance functional of system (1) can be described as

$$
\begin{aligned}
& J_{\left(\tilde{f}_{c}, \tilde{f}_{d}\right)}^{t_{\tilde{f}}^{\tilde{s}}}\left(x_{0}, u_{c}, u_{d}\right)=\int_{t_{0}}^{t_{\tilde{f}}} L_{c}\left(t, x(t), u_{c}(t)\right) \mathrm{d} t \\
& \quad+\sum_{t_{0} \leqslant t_{k}<t_{\tilde{f}}} L_{d}\left(t_{k}, x_{k}, u_{d}\left(t_{k}\right)\right)
\end{aligned}
$$

where $x_{k}=x\left(t_{k}\right), x\left(t_{f}\right)=x_{f}$, where $t_{0}<t_{f}<\infty$ or $t_{f}=$ $\infty ; L_{c}: \mathbf{R}_{+} \times \mathbf{R}^{n} \times \mathbf{R}^{n_{c}} \rightarrow \mathbf{R}$ and $L_{d}: \mathbf{R}_{+} \times \mathbf{R}^{n} \times \mathbf{R}^{n_{d}} \rightarrow \mathbf{R}$ are given.

The optimal robust control problem of (1) with hybrid performance functional (2) is to design the state feedback control law $\left(u_{c}(t), u_{d}\left(t_{k}\right)\right)=\left(\phi_{c}\left(x(t), \phi_{d}\left(x_{k}\right)\right)\right.$, where $\phi_{c}$ : $\mathbf{R}^{n} \rightarrow \mathbf{R}^{n_{c}}, \phi_{d}: \mathbf{R}^{n} \rightarrow \mathbf{R}^{n_{d}}$ with $\phi_{c}(0)=0, \phi_{d}(0)=0$, such that the closed-loop system is robustly asymptotically 
stable, and the bounds of $J_{\left(\tilde{f}_{c}, \tilde{f}_{d}\right)}^{t_{\tilde{\tilde{f}}}}\left(x_{0}, \phi_{c}(x), \phi_{d}(x)\right)$ can be estimated. Moreover, $\left(u_{c}(t), u_{d}\left(t_{k}\right)\right)=\left(\phi_{c}\left(x(t), \phi_{d}\left(x_{k}\right)\right)\right.$ can minimizes the $J_{\left(\tilde{f}_{c 0}, \tilde{f}_{d 0_{k}}\right)}^{t_{\tilde{f}}}\left(x_{0}, u_{c}, u_{d}\right)$, which is the hybrid performance functional of the nominal system of (1). To proceed, some more preliminaries are needed.

Definition 2.1 (1) $X(t) \in \mathbf{R}^{n \times n}$ is said to be a positive definite matrix function if for any $t \in \mathbf{R}_{+}, X(t)$ is a positive definite matrix.

(2) $X(t) \in \mathbf{R}^{n \times n}$ is said to be a positive definite matrix function bounded from above if $X(t) \in \mathbf{R}^{n \times n}$ is a positive definite matrix function and there exists a positive real number $M>0$ such that

$$
\lambda_{\max }(X(t)) \leqslant M, t \in \mathbf{R}_{+}
$$

where $\lambda_{\max }(\cdot)$ is the maximum eigenvalue.

(3) $X(t) \in \mathbf{R}^{n \times n}$ is said to be a uniformly positive definite matrix function if $X(t) \in \mathbf{R}^{n \times n}$ is a positive definite matrix function and there exists a positive real number $m>0$ such that

$$
\lambda_{\min }(X(t)) \geqslant m, t \in \mathbf{R}_{+}
$$

where $\lambda_{\min }(\cdot)$ is the minimum eigenvalue of matrix $(\cdot)$.

Definition 2.2 Let $V: \mathbf{R}_{+} \times \mathbf{R}^{n} \rightarrow \mathbf{R}_{+}$, then $V$ is said to belong to class $\nu_{0}$ if

1) $V$ is continuous in $\left(t_{k-1}, t_{k}\right] \times \mathbf{R}^{n}$ and for each $x \in$ $\mathbf{R}^{n}, t \in\left(t_{k-1}, t_{k}\right], k \in \mathbf{N}$,

$$
\lim _{(t, y) \rightarrow\left(t_{k-1}^{+}, x\right)} V(t, y)=V\left(t_{k-1}^{+}, x\right)
$$

exists;

2) $V$ is locally Lipschitzian in $x$

Definition 2.4 Under hybrid control $\left(u_{c}(t), u_{d}\left(t_{k}\right)\right)=$ $\left(\phi_{c}\left(x(t), \phi_{d}\left(x_{k}\right)\right)\right.$, the uncertain impulsive system (1) is called robustly stable, robustly asymptotically stable, and robustly exponentially stable, respectively, if for any $\tilde{f}_{c} \in \tilde{F}_{c}, \tilde{f}_{d} \in F_{d}$, the equilibrium $x=0$ of (1) is stable, asymptotic stable, and exponential stable, respectively.

\section{MAIN RESULTS}

In this section, we apply the results developed in [9] to analyze the hybrid performance functional $J_{\left(f_{c}, f_{d}\right)}^{t_{f}}\left(x_{0}\right)$ and robust stability criteria for system (1).

Theorem 3.1 Assume that there exist functions $\Gamma_{c}: \mathbf{R}_{+} \times$ $\mathbf{R}^{n} \times \mathbf{R}^{n_{c}} \rightarrow \mathbf{R}, \Gamma_{d}: \mathbf{R}_{+} \times \mathbf{R}^{n} \times \mathbf{R}^{n_{d}} \rightarrow \mathbf{R}, V \in \nu_{0}$, where $V$ is $C^{1}$ function, and $\phi_{c}: \mathbf{R}^{n} \rightarrow \mathbf{R}^{n_{c}}, \phi_{d}: \mathbf{R}^{n} \rightarrow \mathbf{R}^{n_{d}}$ with $\phi_{c}(0)=0, \phi_{d}(0)=0$, such that

(i) there exist $a, b \in \mathcal{K}$, such that for $(t, x) \in \mathbf{R}_{+} \times \mathbf{R}^{n}$,

$$
b(\|x\|) \leqslant V(t, x) \leqslant a(\|x\|)
$$

(ii) for all $t \in\left(t_{k-1}, t_{k}\right]$,

$$
\begin{aligned}
& V_{x}(t, x) \tilde{f}_{c}\left(t, x, \phi_{c}(x)\right) \leqslant V_{x}(t, x) \tilde{f}_{c 0}\left(t, x, \phi_{c}(x)\right) \\
& +\Gamma_{c}\left(t, x, \phi_{c}(x)\right)
\end{aligned}
$$

(iii) there exist $c \in \mathcal{K}, p \in P C$ such that for all $t \in$ $\left(t_{k-1}, t_{k}\right]$

$$
\begin{aligned}
& V_{t}(t, x)+V_{x}(t, x) \tilde{f}_{c 0}\left(t, x, \phi_{c}(x)\right) \\
& +\Gamma_{c}\left(t, x, \phi_{c}(x)\right) \leqslant p(t) c(V(t, x(t)))
\end{aligned}
$$

(iv) there exist $\psi_{k} \in \mathcal{K}_{0}, k \in \mathbf{N}$, such that

$$
\begin{aligned}
& V\left(t_{k}, x_{k}+\tilde{f}_{d}\left(t_{k}, x_{k}, \phi_{d}\left(x_{k}\right)\right)\right) \\
& \leqslant V\left(t_{k}, x_{k}+\tilde{f}_{d 0}\left(t_{k}, x_{k}, \phi_{d}\left(x_{k}\right)\right)\right) \\
& +\Gamma_{d}\left(t_{k}, x_{k}, \phi_{d}\left(x_{k}\right)\right) \leqslant \psi_{k}\left(V\left(t_{k}, x_{k}\right)\right)
\end{aligned}
$$

(v) there exists a constant $\sigma>0$ such that for all $z \in(0, \sigma)$

$$
\int_{t_{k}}^{t_{k+1}} p(s) \mathrm{d} s+\int_{z}^{\psi_{k}(z)} \frac{\mathrm{d} s}{c(s)} \leqslant-r_{k}
$$

where $r_{k} \geqslant 0$ and $\sum_{k=1}^{\infty} r_{k}=+\infty$;

(vi) for any $k \in \mathbf{N}$,

$$
\begin{aligned}
& H_{c}\left(t, x, \phi_{c}(x)\right)=0, \text { for all } t \in\left(t_{k-1}, t_{k}\right] \\
& H_{d}\left(t_{k}, x_{k}, \phi_{d}\left(x_{k}\right)\right)=0, \quad k \in \mathbf{N} \\
& H_{c}\left(t, x, u_{c}\right) \geqslant 0, \quad \text { for all } u_{c} \in U_{c} \\
& H_{d}\left(t, x, u_{d}\right) \geqslant 0, \quad \text { for all } u_{d} \in U_{d}
\end{aligned}
$$

where

$$
\begin{aligned}
& H_{c}\left(t, x, u_{c}\right)=L_{d}\left(t, x, u_{c}\right)+V_{t}(t, x) \\
& +V_{x}(t, x) \tilde{f}_{c 0}\left(t, x, u_{c}\right)+\Gamma_{c}\left(t, x, u_{c}\right) \\
& H_{d}\left(t, x, u_{d}\right)=V\left(t, x+\tilde{f}_{d 0}\left(t, x, u_{d}\right)\right) \\
& +L_{d}\left(t, x, u_{d}\right)-V(t, x)+\Gamma_{d}\left(t, x, u_{d}\right)
\end{aligned}
$$

Then, with the state feedback control law $\left(u_{c}(t), u_{d}\left(t_{k}\right)=\right.$ $\left(\phi_{c}(x(t)), \phi_{d}\left(x_{k}\right)\right), t \geqslant t_{0}, k \in \mathbf{N}$, the zero solution $x(t)=$ 0 of the closed-loop system (1) is robustly asymptotically stable for all $\left(\tilde{f}_{c}, \tilde{f}_{d}\right) \in \tilde{F}$, and

$$
\begin{aligned}
& \sup _{\left(\tilde{f}_{c}, \tilde{f}_{d}\right) \in \tilde{F}} J_{\left(\tilde{f}_{c}, \tilde{f}_{d}\right)}^{t_{\tilde{f}}}\left(x_{0}, \phi_{c}(x), \phi_{d}(x)\right) \\
& \leqslant I_{\left(\tilde{f}_{c 0}, \tilde{f}_{d 0}\right)}^{t_{\tilde{f}}}\left(x_{0}, \phi_{c}(x), \phi_{d}(x)\right) \leqslant V\left(t_{0}, x_{0}\right)
\end{aligned}
$$

Specially,

$$
\begin{aligned}
& \sup _{\left(\tilde{f}_{c}, \tilde{f}_{d}\right) \in F} J_{\left(\tilde{f}_{c}, \tilde{f}_{d}\right)}^{\infty}\left(x_{0}, \phi_{c}(x), \phi_{d}(x)\right) \\
& \leqslant I_{\left(\tilde{f}_{c 0}, \tilde{f}_{d 0}\right)}^{\infty}\left(x_{0}, \phi_{c}(x), \phi_{d}(x)\right)=V\left(t_{0}, x_{0}\right)
\end{aligned}
$$

where

$$
\begin{aligned}
& I_{\left(\tilde{f}_{c 0}, \tilde{f}_{d 0}\right)}^{t_{\tilde{f}}}\left(x_{0}, u_{c}, u_{d}\right)=\int_{t_{0}}^{t_{\tilde{f}}}\left[L_{c}\left(t, x(t), u_{c}(t)\right)\right. \\
& \left.+\Gamma_{c}\left(t, x(t), u_{c}(t)\right)\right] \mathrm{d} t+\sum_{t_{0} \leqslant t_{k}<t_{\tilde{f}}}\left[L _ { d } \left(t_{k}\right.\right. \\
& \left.\left.x\left(t_{k}\right), u_{d}\left(t_{k}\right)\right)+\Gamma_{d}\left(t_{k}, x_{k}, u_{d}\left(t_{k}\right)\right)\right]
\end{aligned}
$$

where $t_{\tilde{f}}<\infty$ or $t_{\tilde{f}}=\infty$, and in (18)-(19), $x(t), t \neq t_{0}$, is a solution to system (1) with $\left(\tilde{f}_{c}, \tilde{f}_{d}\right)=\left(\tilde{f}_{c 0}, \tilde{f}_{d 0}\right)$.

Moreover, the hybrid feedback control law $\left(u_{c}(t), u_{d}\left(t_{k}\right)\right)=$ $\left(\phi_{c}(x(t)), \phi_{d}\left(x_{k}\right)\right), t \geqslant t_{0}, k \in N$, minimizes $I_{\left(\tilde{f}_{c 0}, \tilde{f}_{d 0}\right)}^{\infty}\left(x_{0}, u_{c}, u_{d}\right)$ in sense that

$$
\begin{aligned}
& I_{\left(\tilde{f}_{c 0}, \tilde{f}_{d 0}\right)}^{\infty}\left(x_{0}, \phi_{c}(x), \phi_{d}(x)\right) \\
& =\min _{\left(u_{c}, u_{d}\right) \in\left(U_{c}, U_{d}\right)} I_{\left(\tilde{f}_{c 0}, \tilde{f}_{d 0}\right)}^{\infty}\left(x_{0}, u_{c}, u_{d}\right)
\end{aligned}
$$


Proof At first, we show system (1) is robustly asymptotically stable. Let $x(t), t \neq t_{0}$ be the solution to system (1). Then, by conditions (ii)-(iii), we get

$$
\begin{aligned}
& D^{+} V(t, x(t))=V_{t}(t, x)+V_{x}(t, x) f_{c}\left(t, x, \phi_{c}(x)\right) \\
& \leqslant V_{t}(t, x)+V_{x}(t, x) f_{c 0}\left(t, x, \phi_{c}(x)\right) \\
& \quad+\Gamma_{c}\left(t, x, \phi_{c}(x)\right) \\
& \leqslant p(t) c(V(t, x(t))), \quad t \in\left(t_{k-1}, t_{k}\right], k \in \mathbf{N}
\end{aligned}
$$

and from condition (iv),

$$
V\left(t_{k}, x_{k}+f_{d}\left(t_{k}, x_{k}, \phi_{d}\left(x_{k}\right)\right)\right) \leqslant \psi_{k}\left(V\left(t_{k}, x_{k}\right)\right)
$$

Thus, from (21)-(22) and conditions (i) and (v), we obtain that all conditions of Theorem 3.1 in [9] are satisfied and hence, under the hybrid control law $\left(u_{c}(t), u_{d}\left(t_{k}\right)=\left(\phi_{c}(x(t)), \phi_{d}\left(x_{k}\right)\right), t \geqslant t_{0}, k \in \mathbf{N}\right.$, (1) is robustly asymptotically stable for all $\left(\tilde{f}_{c}, \tilde{f}_{d}\right) \in \tilde{F}$.

Secondly, we show estimations (17)-(18) hold.

By conditions (ii) and (vi) ((11)), we get

$$
\begin{aligned}
& L_{c}\left(t, x, \phi_{c}(x)\right)=-D^{+} V(t, x(t))+L_{c}\left(t, x, \phi_{c}(x)\right) \\
& \quad+V_{t}(t, x)+V_{x}(t, x) \tilde{f}_{c}\left(t, x, \phi_{c}(x)\right) \\
& \leqslant-D^{+} V(t, x(t))+H_{c}\left(t, x, \phi_{c}(x)\right) \\
& =-D^{+} V(t, x(t))
\end{aligned}
$$

By conditions (iv) and (vi)((12)), we have

$$
\begin{aligned}
& L_{d}\left(t_{k}, x_{k}, \phi_{d}\left(x_{k}\right)\right) \\
& =-\triangle V\left(t_{k}, x_{k}\right)+L_{d}\left(t_{k}, x_{k}, \phi_{d}\left(x_{k}\right)\right) \\
& +V\left(t_{k}, x_{k}+\tilde{f}_{d}\left(t_{k}, x_{k}, \phi_{d}\left(x_{k}\right)\right)\right)-V\left(t_{k}, x_{k}\right) \\
& \leqslant-\triangle V\left(t_{k}, x_{k}\right)+H_{d}\left(t_{k}, x_{k}, \phi_{d}\left(x_{k}\right)\right) \\
& =-\triangle V\left(t_{k}, x_{k}\right)
\end{aligned}
$$

Now, integrating over the interval $\left[t_{0}, t_{f}\right)$ with $\{k \in \mathbf{N}$ : $\left.t_{0} \leqslant t_{k}<t_{f}\right\}=\{1,2, \cdots m\}$, by (23) and (24), we have

$$
\begin{aligned}
J_{\left(\tilde{f}_{c}, \tilde{f}_{d}\right)}^{t_{f}} & \left(x_{0}, \phi_{c}, \phi_{d}\right) \\
= & \sum_{i=0}^{m-1} \int_{t_{i}^{+}}^{t_{i+1}} L_{c}\left(t, x(t), \phi_{c}(x(t))\right) \mathrm{d} t \\
& +\int_{t_{m}^{+}}^{t_{f}} L_{c}\left(t, x, \phi_{c}(x)\right) \mathrm{d} t+\sum_{i=1}^{m} L_{d}\left(t_{i}, x_{i}, \phi_{d}\left(x_{i}\right)\right) \\
\leqslant & -\sum_{i=0}^{m-1} \int_{t_{i}^{+}}^{t_{i+1}} D^{+} V(t, x) \mathrm{d} t-\int_{t_{m}^{+}}^{t_{f}} D^{+} V(t, x) \mathrm{d} t \\
& -\sum_{i=1}^{m} \triangle V\left(t_{i}, x_{i}\right) \\
= & -\left(V\left(t_{1}, x_{1}\right)-V\left(t_{0}, x_{0}\right)+V\left(t_{2}, x_{2}\right)\right. \\
& -V\left(t_{1}, x_{1}+f_{d}\left(t_{1}, x_{1}\right)\right)+\cdots+V\left(t_{m}, x_{m}\right) \\
& -V\left(t_{m-1}, x_{m-1}+f_{d}\left(t_{m-1}, x_{m-1}\right)\right) \\
& -\left(V\left(t_{f}, x_{f}\right)-V\left(t_{m}, x_{m}+f_{d}\left(t_{m}, x_{m}\right)\right)\right. \\
& -\sum_{i=1}^{m} \triangle V\left(t_{i}, x_{i}\right)=V\left(t_{0}, x_{0}\right)-V\left(t_{f}, x_{f}\right)
\end{aligned}
$$

Moreover, if $x(t)$ is a solution to (1) with $\left(\tilde{f}_{c}, \tilde{f}_{d}\right)=$ $\left(\tilde{f}_{c 0}, \tilde{f}_{d 0}\right)$, then, by condition (ii) and (11), for all $t \in$ $\left(t_{k-1}, t_{k}\right]$, we get

$$
\begin{aligned}
& L_{c}\left(t, x, \phi_{c}(x)\right)+\Gamma_{c}\left(t, x(t), \phi_{c}(x)\right) \\
& =-D^{+} V(t, x(t))
\end{aligned}
$$

Similarly, the condition (iv) and (12) implies that

$$
\begin{aligned}
& L_{d}\left(t_{k}, x_{k}, \phi_{d}\left(x_{k}\right)\right)+\Gamma_{d}\left(t_{k}, x_{k}, \phi_{d}\left(x_{k}\right)\right) \\
& =-\triangle V\left(t_{k}, x_{k}\right)
\end{aligned}
$$

Hence, integrating over the interval $\left[t_{0}, t_{f}\right)$ with $\{k \in \mathbf{N}$ : $\left.t_{0} \leqslant t_{k}<t_{f}\right\}=\{1,2, \cdots m\}$, by (26)-(27), we have

$$
\begin{aligned}
& I_{\left(\tilde{f}_{c 0}, \tilde{f}_{d 0}\right)}^{t_{f}}\left(x_{0}, \phi_{c}(x), \phi_{d}(x)\right) \\
& =\sum_{i=0}^{m-1} \int_{t_{i}^{+}}^{t_{i+1}}\left[L_{c}\left(t, x, \phi_{c}(x)\right)+\Gamma_{c}\left(t, x, \phi_{c}(x)\right)\right] \mathrm{d} t \\
& \quad+\int_{t_{m}^{+}}^{t_{f}}\left[L_{c}\left(t, x, \phi_{c}(x)\right)+\Gamma_{c}\left(t, x, \phi_{c}(x)\right)\right] \mathrm{d} t \\
& \quad+\sum_{i=1}^{m}\left[L_{d}\left(t_{i}, x\left(t_{i}\right), \phi_{d}\left(x_{i}\right)\right)+\Gamma_{d}\left(t_{i}, x_{i}, \phi_{d}\left(x_{i}\right)\right)\right] \\
& =-\sum_{i=0}^{m-1} \int_{t_{i}^{+}}^{t_{i+1}} D^{+} V(t, x(t)) \mathrm{d} t \\
& \quad-\int_{t_{m}^{+}}^{t_{f}} D^{+} V(t, x(t)) \mathrm{d} t-\sum_{i=1}^{m} \triangle V\left(t_{i}, x_{i}\right) \\
& =V\left(t_{0}, x_{0}\right)-V\left(t_{f}, x_{f}\right)
\end{aligned}
$$

Thus, by $V\left(t_{f}, x_{f}\right) \geqslant 0$ and (25) and (28), we obtain that (17) holds. Moreover, by the asymptotic stability, which means that $\lim _{t_{f} \rightarrow \infty} V\left(t_{f}, x_{f}\right)=0$, we get that (18) also holds. Finally, we need to prove that the hybrid control law $\left(u_{c}(t), u_{d}\left(t_{k}\right)\right)=\left(\phi_{c}(x(t)), \phi_{d}\left(x\left(t_{k}\right)\right)\right), t \geqslant t_{0}, k \in \mathbf{N}$, minimizes $I_{\left(\tilde{f}_{c 0}, \tilde{f}_{d 0}\right)}^{\infty}\left(x_{0}, u_{c}, u_{d}\right)$ in sense of (20).

Let $\left(\tilde{f}_{c}, \tilde{f}_{d}\right)=\left(\tilde{f}_{c 0}, \tilde{f}_{d 0}\right)$, then, by (15), for all $t \in$ $\left(t_{k-1}, t_{k}\right], k \in \mathbf{N}$,

$$
\begin{aligned}
& L_{c}\left(t, x(t), u_{c}(t)\right)+\Gamma_{c}\left(t, x(t), u_{c}(t)\right) \\
& =-D^{+} V(t, x(t))+H_{c}\left(t, x(t), u_{c}(t)\right)
\end{aligned}
$$

and by (16), for any $k \in \mathbf{N}$,

$$
\begin{aligned}
& L_{d}\left(t_{k}, x_{k}, u_{d}\left(t_{k}\right)\right)+\Gamma_{d}\left(t_{k}, x_{k}, u_{d}\left(t_{k}\right)\right) \\
& =-\triangle V\left(t_{k}, x_{k}\right)+H_{d}\left(t_{k}, x_{k}, u_{d}\left(t_{k}\right)\right)
\end{aligned}
$$

Thus, using (29) and (30), and the fact that system (1) with $\left(\tilde{f}_{c}, \tilde{f}_{d}\right)=\left(\tilde{f}_{c 0}, \tilde{f}_{d 0}\right)$ is asymptotic stable, we have

$$
\begin{aligned}
& I_{\left(\tilde{f}_{c 0}, \tilde{f}_{d 0}\right)}^{\infty}\left(x_{0}, u_{c}, u_{d}\right)=\int_{t_{0}}^{\infty}\left[-D^{+} V(t, x(t))\right. \\
& \left.+H_{c}\left(t, x(t), u_{c}(t)\right)\right] \mathrm{d} t \\
& +\sum_{k=1}^{\infty}\left[-\triangle V\left(t_{k}, x_{k}\right)+H_{d}\left(t_{k}, x_{k}, u_{d}\left(t_{k}\right)\right)\right] \\
= & -\lim _{t \rightarrow \infty} V(t, x(t))+V\left(t_{0}, x_{0}\right) \\
+ & \int_{t_{0}}^{\infty} H_{c}\left(t, x(t), u_{c}(t)\right) \mathrm{d} t+\sum_{k=1}^{\infty} H_{d}\left(t_{k}, x_{k}, u_{d}\left(t_{k}\right)\right) \\
\geqslant & V\left(t_{0}, x_{0}\right)=I_{\left(\tilde{f}_{c 0}, \tilde{f}_{d 0}\right)}^{\infty}\left(x_{0}, \phi_{c}(x), \phi_{d}(x)\right)
\end{aligned}
$$


Hence, by (31), the hybrid feedback control law $\left(u_{c}(t), u_{d}\left(t_{k}\right)\right)=\left(\phi_{c}(x(t)), \phi_{d}\left(x_{k}\right)\right), t \geqslant t_{0}, k \in \mathbf{N}$, minimizes $I_{\left(\tilde{f}_{c 0}, \tilde{f}_{d 0}\right)}^{\infty}\left(x_{0}, u_{c}, u_{d}\right)$ in sense (20). The proof is complete.

Remark 3.1 (1') By Theorem 3.2 in [9], in Theorem 3.1, if $p(t)$ is replaced by $-p(t)$, then the results of Theorem 3.1 still hold.

(2') By (11)-(12), if nonnegative functions $H_{c}\left(t, x, u_{c}\right)$, $H_{d}\left(t, x, u_{d}\right)$ are continuously differentiable in $u_{c}$ and $u_{d}$, respectively, then,

$$
\left.\frac{\partial H_{c}\left(t, x, u_{c}\right)}{\partial u_{c}}\right|_{u_{c}=\phi_{c}(x)}=0,\left.\frac{\partial H_{d}\left(t, x, u_{d}\right)}{\partial u_{d}}\right|_{u_{d}=\phi_{d}(x)}=0
$$

which can be used to derive the hybrid state feedback control law $\left(u_{c}, u_{d}\right)=\left(\phi_{c}(x), \phi_{d}(x)\right)$.

$\left(3^{\prime}\right)$ From the proof of Theorem 3.1, we get that $D^{+} V(t, x) \leqslant p(t) c(V(t, x))$ for any $t \neq t_{k}$. While in [6], it needs that $D^{+} V(t, x) \leqslant 0$ for any $t \neq t_{k}$. Since $p(t) \geqslant 0$, the results of Theorem 3.1 is less conservative than that in [6].

\section{SPECIALIZATION TO LINEAR IMPULSIVE SYSTEMS}

In this section, we specialize Theorem 3.1 to interval linear impulsive system.

Consider interval linear impulsive system in form:

$$
\begin{aligned}
& \dot{x}=\left(A_{c}(t)+B_{c}(t)\right) x+D_{c}(t) u_{c}(t), \quad t \neq t_{k} \\
& \Delta x=\left(A_{d_{k}}(t)+B_{d_{k}}(t)\right) x+D_{d_{k}} u_{d}(t), \quad t=t_{k} \\
& x\left(t_{0}^{+}\right)=x_{0}, \quad k \in \mathbf{N}
\end{aligned}
$$

with the hybrid quadratic performance functional:

$$
\begin{aligned}
& J_{\left(\tilde{A}_{c}, \tilde{A}_{d_{k}}\right)}^{t_{f}}\left(x_{0}, u_{c}, u_{d}\right)=\int_{t_{0}}^{t_{f}}\left[x^{\mathrm{T}}(t) R_{c}(t) x(t)\right. \\
& \left.\quad+u_{c}^{\mathrm{T}}(t) S_{c}(t) u_{c}(t)\right] \mathrm{d} t+\sum_{t_{0} \leqslant t_{k}<t_{f}}\left[x_{k}^{\mathrm{T}} R_{d} x_{k}\right. \\
& \left.\quad+u_{d}^{\mathrm{T}}\left(t_{k}\right) S_{d}\left(t_{k}\right) u_{d}\left(t_{k}\right)\right]
\end{aligned}
$$

where $A_{c}(t), A_{d_{k}}(t) \in \mathbf{R}^{n \times n}, D_{c}(t) \in \mathbf{R}^{n \times n_{c}}, D_{d_{k}}(t) \in$ $\mathbf{R}^{n \times n_{d}}, \quad S_{c}(t) \in \mathrm{P}^{n_{c} \times n_{c}}, \quad S_{d}(t) \in \mathbf{N}^{n_{d} \times n_{d}}$, $R_{c}(t) \in \mathrm{P}^{n \times n}$, and $R_{d} \in \mathbf{N}^{n \times n}$, are known matrices, while $B_{c}(t), B_{d_{k}}(t), k \in \mathbf{N}$ are interval matrices: $B_{c}(t) \in N\left[P_{c}(t), Q_{c}(t)\right]=\left\{B_{c}(t) \in \mathbf{R}^{n \times n}\right.$ : $B_{c}(t)=\left(b_{c}(t)_{i j}\right)_{n \times n}, p_{c}(t)_{i j} \leqslant b_{c}(t)_{i j} \leqslant q_{c}(t)_{i j} ; i, j=$ $1,2, \ldots, n\}, \quad B_{d_{k}}(t) \in N\left[P_{d_{k}}(t), Q_{d_{k}}(t)\right]$, where $P_{c}(t)=\left(p_{c}(t)_{i j}\right)_{n \times n}, P_{d_{k}}(t)=\left(p_{d_{k}}(t)_{i j}\right)_{n \times n}, Q_{c}(t)=$ $\left(q_{c}(t)_{i j}\right)_{n \times n}, Q_{d_{k}}(t)=\left(q_{d_{k}}(t)_{i j}\right)_{n \times n}, k \in \mathbf{N}$, are known matrices.

By Lemma 3.1 in [9], for any interval matrix $B(t) \in$ $N[P(t), Q(t)]$, where $P=\left(p(t)_{i j}\right)_{n \times n}$ and $Q=$ $\left(q(t)_{i j}\right)_{n \times n}$ are known matrices, then, we have the representation of $B(t)$ as follows:

$$
B(t)=B(t)_{0}+E(t) \Sigma(t) F(t)
$$

where $B(t)_{0}=\frac{1}{2}(P(t)+Q(t)), \quad H(t)=\left(h(t)_{i j}\right)_{n \times n}=$ $\frac{1}{2}(Q(t)-P(t)), \Sigma(t) \in \Sigma^{*}=\left\{\Sigma(t) \in \mathbf{R}^{n^{2} \times n^{2}}: \Sigma(t)=\right.$ $\operatorname{diag}\left(\varepsilon(t)_{11,}, \cdots, \varepsilon(t)_{1 n}, \cdots, \varepsilon(t)_{n 1}, \cdots\right.$, $\left.\left.\varepsilon(t)_{n n}\right), \quad\left|\varepsilon(t)_{i j}\right| \leqslant 1 ; i, j=1,2, \cdots, n\right\}$, and $E(t) E(t)^{\mathrm{T}}=\operatorname{diag}\left(\sum_{j=1}^{n} h(t)_{1 j} \cdots \sum_{j=1}^{n} h(t)_{n j}\right)$, $F(t)^{\mathrm{T}} F(t)=\operatorname{diag}\left(\sum_{j=1}^{n} h(t)_{j 1} \cdots \sum_{j=1}^{n} h(t)_{j n}\right)$.

In the following, denote: $A_{c}(t)+B_{c}(t)=$ $A_{c 0}(t)+E_{c}(t) \Sigma_{c}(t) F_{c}(t)$ and $A_{d_{k}}(t)+B_{d_{k}}(t)=$ $A_{d_{0}}(t)+E_{d_{k}}(t) \Sigma_{d_{k}}(t) F_{d_{k}}(t), \Sigma_{d_{k}} \in \Sigma^{*}, k \in \mathbf{N}$.

Theorem 4.1 Assume that there exist scalar functions $\lambda_{k}(t)>0, \alpha(t)>0, k \in \mathbf{N}$, and a uniformly positive definite matrix function bounded from above $X(t)$ such that

(i) $X(t)$ is differentiable at $t \neq t_{k}$ and for all $t \in$ $\left(t_{k-1}, t_{k}\right], k \in \mathbf{N}$, the Riccati inequality holds:

$$
\begin{aligned}
& \dot{X}+X\left(A_{c 0}+D_{c} K_{c}\right)+\left(A_{c 0}+D_{c} K_{c}\right)^{\mathrm{T}} X \\
& +2 \lambda_{k}^{2} X E_{c} E_{c}^{\mathrm{T}} X+\frac{1}{2 \lambda_{k}^{2}} F_{c}^{\mathrm{T}} F_{c}<-\alpha X
\end{aligned}
$$

where $K_{c}=K_{c}(t)=-S_{c}^{-1}(t) D_{c}^{\mathrm{T}}(t) X(t), \lambda_{k}=\lambda_{k}(t)$, and $\alpha=\alpha(t)$;

(ii)

$$
\begin{aligned}
& R_{c}+K_{c}^{\mathrm{T}} S_{c} K_{c}+\dot{X}+X\left(A_{c 0}+D_{c} K_{c}\right)+\left(A_{c 0}+\right. \\
& \left.D_{c} K_{c}\right)^{\mathrm{T}} X+2 \lambda_{k}^{2} X E_{c} E_{c}^{\mathrm{T}} X+\frac{1}{2 \lambda_{k}^{2}} F_{c}^{\mathrm{T}} F_{c}=0
\end{aligned}
$$

(iii) there exist some $r_{k} \geqslant 0$ with $\sum_{k=1}^{n} r_{k}=+\infty$, and $\epsilon_{k}>0, k \in \mathbf{N}$ such that for all $k \in \mathbf{N}$,

$$
-\int_{t_{k}}^{t_{k+1}} \alpha(s) \mathrm{d} s+\ln \beta_{k} \leqslant-r_{k}
$$

where

$\beta_{k}=\lambda_{\max }\left\{X_{k}^{-1}\left[\left(I+A_{d 0_{k}}+D_{d_{k}} K_{d_{k}}\right)^{\mathrm{T}}\left[X_{k}+\right.\right.\right.$ $\left.\epsilon_{k}^{-1} X_{k} E_{d_{k}} E_{d_{k}}^{\mathrm{T}} X_{k}\right]\left(I+A_{d_{0}}+D_{d_{k}} K_{d_{k}}\right)+\left[\epsilon_{k}+\right.$ $\left.\left.\left.\lambda_{\max }\left(E_{d_{k}}^{\mathrm{T}} X_{k} E_{d_{k}}\right)\right] F_{d_{k}}^{\mathrm{T}} F_{d_{k}}\right]\right\}$

and where, $X_{k}=X\left(t_{k}\right), E_{d_{k}}=E_{d_{k}}\left(t_{k}\right), F_{d_{k}}=F_{d_{k}}\left(t_{k}\right)$, $S_{d_{k}}=S_{d}\left(t_{k}\right)$, and $D_{d_{k}}=D_{d_{k}}\left(t_{k}\right), K_{d_{k}}=K_{d}\left(t_{k}\right)=$ $-S_{d_{k}}^{-1} D_{d_{k}}^{\mathrm{T}} X_{k}\left(I+A_{d 0_{k}}\right)$

(iv)

$$
\begin{aligned}
& R_{d}+K_{d_{k}}^{\mathrm{T}} S_{d_{k}} K_{d_{k}}-X_{k}+\left(I+A_{d_{0}}+D_{d_{k}} K_{d_{k}}\right)^{\mathrm{T}} \\
& \cdot\left[X_{k}+\epsilon_{k}^{-1} X_{k} E_{d_{k}} E_{d_{k}}^{\mathrm{T}} X_{k}\right]\left(I+A_{d_{0}}+D_{d_{k}} K_{d_{k}}\right) \\
& +\left[\epsilon_{k}+\lambda_{\max }\left(E_{d_{k}}^{\mathrm{T}} X_{k} E_{d_{k}}\right)\right] F_{d_{k}}^{\mathrm{T}} F_{d_{k}}=0
\end{aligned}
$$

Then, with the state feedback control law

$$
\left(u_{c}, u_{d}\right)=\left(\phi_{c}(x), \phi_{d}(x)\right)=\left(K_{c} x, K_{d} x\right)
$$

the zero solution $x(t)=0$ of the closed-loop system (32) and (39) is robustly asymptotically stable for all $\left(B_{c}, B_{d_{k}}\right) \in$ $\Sigma=\Sigma_{c}^{*} \times \Sigma_{d}^{*}$, and

$$
\begin{aligned}
& \sup _{\left(\Sigma_{c}, \Sigma_{d_{k}}\right) \in \Sigma} J_{\left(\tilde{A}_{c}, \tilde{A}_{d_{k}}\right)}^{t_{f}}\left(x_{0}, u_{c}, u_{d}\right) \\
& \leqslant I_{\left(A_{c 0}, A_{d 0_{k}}\right)}^{t_{f}}\left(x_{0}, \phi_{c}(x), \phi_{d}(x)\right) \leqslant x_{0}^{\mathrm{T}} X_{0} x_{0}
\end{aligned}
$$

Specially,

$$
\begin{aligned}
& \sup _{\left(\Sigma_{c}, \Sigma_{d_{k}}\right) \in \Sigma} J_{\left(\tilde{A}_{c}, \tilde{A}_{d_{k}}\right)}^{\infty}\left(x_{0}, u_{c}, u_{d}\right) \\
& \leqslant I_{\left(A_{c 0}, A_{d 0_{k}}\right)}^{\infty}\left(x_{0}, \phi_{c}(x), \phi_{d}(x)\right)=x_{0}^{\mathrm{T}} X_{0} x_{0}
\end{aligned}
$$


where $X_{0}=X\left(t_{0}\right)$, and,

$$
\begin{aligned}
& I_{\left(A_{c 0}, A_{d 0_{k}}\right)}^{t_{f}}\left(x_{0}, u_{c}, u_{d}\right)=\int_{t_{0}}^{t_{f}}\left(x ^ { \mathrm { T } } ( t ) \left[R_{c}(t)\right.\right. \\
& +2 \lambda_{k}^{2}(t) X(t) E_{c}(t) E_{c}^{\mathrm{T}}(t) X(t) \\
& \left.\left.+\frac{1}{2 \lambda_{k}^{2}(t)} F_{c}^{\mathrm{T}}(t) F_{c}(t)\right] x(t)+u_{c}^{\mathrm{T}}(t) S_{c}(t) u_{c}(t)\right) \mathrm{d} t \\
& +\sum_{t_{0} \leqslant t_{k}<t_{f}}\left\{x _ { k } \left[R_{d}+\epsilon_{k}^{-1}\left(I+A_{d 0_{k}}\right)^{\mathrm{T}} X_{k} E_{d_{k}}\right.\right. \\
& \cdot E_{d_{k}}^{\mathrm{T}} X_{k}\left(I+A_{d 0_{k}}\right)+\left[\epsilon_{k}+\lambda_{\max }\left(E_{d_{k}}^{\mathrm{T}} X_{k} E_{d_{k}}\right)\right] \\
& \left.\left.\cdot F_{d_{k}}^{\mathrm{T}} F_{d_{k}}\right] x_{k}+u_{d}^{\mathrm{T}}\left(t_{k}\right) S_{d}\left(t_{k}\right) u_{d}\left(t_{k}\right)\right\}
\end{aligned}
$$

where $t_{f}<\infty$ or $t_{f}=\infty$, and in (42), $x(t), t \neq t_{0}$, is a solution to system (32) with $\left(B_{c}, B_{d_{k}}\right)=(0,0), k \in \mathbf{N}$.

Moreover,

$$
\begin{aligned}
& I_{\left(A_{c 0}, A_{d 0_{k}}\right)}^{\infty}\left(x_{0}, \phi_{c}(x), \phi_{d}(x)\right) \\
& =\min _{\left(u_{c}, u_{d}\right) \in\left(U_{c}, U_{d}\right)} I_{\left(A_{c 0}, A_{d 0_{k}}\right)}^{\infty}\left(x_{0}, u_{c}, u_{d}\right)
\end{aligned}
$$

Proof Let $V(t, x)=x^{\mathrm{T}} X(t) x$. Then, the results of this theorem are the direct consequence of Theorem 3.1 and Remark 3.1 $\left(1^{\prime}\right)$. The detailed is omitted here.

Remark 4.1 By Theorem 3.1 and remark 3.1(1 $\left.1^{\prime}\right)$, if $\alpha(t)$ is replaced by $-\alpha(t)$ in Theorem 4.1 , the results are still true.

\section{CONCLUSIONS}

In this paper, we proposed the optimal robust control problem for uncertain impulsive systems with hybrid performance functional. By employing the Lyapunov function method and algebraic inequalities, we have derived robust stability conditions and the optimal bound of the hybrid performance functional. As the applications, we have investigated robust optimal control problem for the interval linear impulsive systems with quadratic hybrid performance functional. We have obtained the Riccati inequality conditions under which feedback control law can be designed to stabilize asymptotically the interval linear impulsive system and optimal bound of quadratic hybrid performance functional are derived.

\section{REFERENCES}

[1] Lakshmikantham V, Bainov D D, Simeonov P S. Theory of impulsive differential equations. Singapore: World Scientific, 1989.

[2] Bainov D D, Simeonov P S. Systems with impulse effects: Stability theory and applications. New York: Halsted, 1989.
[3] Liu X Z. Stability results for impulsive differential systems with applications to population growth models[J]. Dynamics and stability of systems, 1994, 9: 163-174.

[4] Ye H, Michel A N, Hou L. Stability analysis of systems with impulse effects[J]. IEEE Trans. Automat. Contr., 1998, 43: 1719-1723.

[5] Isidori A. Nonlinear control systems II. London: Springer, 1999.

[6] Haddad W M, Kablar N A, Chellabonia V S. Nonlinear robust control for nonlinear uncertain impulsive dynamical systems[C]. Proc.IEEE Conf. Dec. Contr., Sydney, Australia, 2000: 2959-2964.

[7] Haddad W M, Chellabonia V S, Kablar N A. Nonlinear Impulsive Dynamical Systems Part 2: Feedback Interconnections and Optimality[C]. Proc.IEEE Conf. Dec. Contr., Phoenix, AZ, 1999: 5225-5234.

[8] Liu B, Liu X Z, Liao X X. Stability and robust stability of quasi-linear impulsive hybrid dynamical systems[J]. J. Math. Anal. Appl., 2003, 283: 416-430.

[9] Liu B, Liu X Z, Liao X X. Robust stability of uncertain impulsive dynamical systems[J]. J. Math. Anal. Appl., 2004, 290: 519-533.

[10] Liu B, Liu X Z, Teo K L. Feedback stabilization of dissipative impulsive dynamical systems[J]. Information Sciences, 2007, 177(7): 1663-1672.

[11] Liu B, Liu X Z, Chen G, Wang H. Robust impulsive synchronization of uncertain dynamical networks[J]. IEEE Trans. on Circ. Sys.-I, 2005, 52(7): 1431-1441.

[12] Liu B, Liu X Z, Teo K L, Wang Q. Razumikhin-type theorems on exponential stability of impulsive delay systems[J]. IMA J. Appl. Math., 2006, 71: 47-61.

[13] Sun J, Zhang Y, Wu Q. Less conservation conditions of stability for impulsive control systems[J]. IEEE Trans. on Automatic Control, 2003, 48: 829-831.

[14] Li Z G, Soh Y C, Wen C Y. Robust stability of a class of hybrid nonlinear systems[J]. IEEE Trans. on Automatic Control, 2001, 46(6): 897-903.

[15] Li Z G, Wen C Y, Soh Y C. Analysis and design of impulsive control systems[J]. IEEE Trans. on Automatic Control, 2001, 46(6): 894-897.

[16] Basin M V, Pinsky M A. Stability impulse control of faulted nonlinear systems[J]. IEEE IEEE Transactions on Automatic Control, 1998, 43(11): 1604-1608.

[17] Basin M V, Pinsky M A. Emergency control of unstable behavior of nonlinear systems induced by fault[J]. Mathematical Problems in Engineering, 1998, 4(4): 301-316.

[18] Liu X Z, Shen J. Stability theory of hybrid dynamical systems with time delays[J]. IEEE Trans. on Automatic Control, 2006, 51(4): 620-625.

[19] Guan Z H, Hill D J, Shen X. On hybrid impulsive and switching systems and application to nonlinear control[J]. IEEE Trans. Auto. Control, 2005, 50(7): 1058-62. 